\title{
Influence of the Piezoelectric Ringing on the Temperature of the Beta Barium Borate Crystal
}

\author{
G. Sinkevicius*, A. Baskys and N. Paulauskas \\ Center for Physical Sciences and Technology, Sauletekio al. 5, LT-10221, Vilnius, Lithuania \\ and \\ Vilnius Gediminas Technical University, Naugarduko g. 41, LT-03227, Vilnius, Lithuania \\ (Received February 25, 2018; in final form July 17, 2018)
}

\begin{abstract}
The influence of the piezoelectric ringing on the temperature of the beta barium borate Pockels cell crystal has been investigated. The experimental setup for the investigations was developed. The measurements of the Pockels cell crystal contrast ratio and temperature dependences on the applied high voltage pulse frequency for 10 different $3 \mathrm{~mm} \times 3 \mathrm{~mm} \times 25 \mathrm{~mm}$ beta barium borate Pockels cell crystals were performed. The transients of the beta barium borate Pockels cell temperature were measured at various high voltage pulse frequencies. Two parameters were recorded: the contrast ratio and the crystal temperature. The frequencies, at which the temperature increase caused by the piezoelectric ringing occurs, were determined. It has been shown that the contrast ratio of the beta barium borate Pockels cell crystal does not depend on the temperature. Additionally, the investigation of the deuterated potassium dihydrogen phosphate Pockels cell crystal ringing phenomenon was performed. It has been shown that from the point of view of the contrast ratio temperature stability Pockels cells based on the beta barium borate crystals are more preferable.
\end{abstract}

DOI: 10.12693/APhysPolA.134.535

PACS/topics: 78.20.Jq, 77.84.-s, 78.20.Hp, 42.70.Hj, 42.60.Gd, 42.65.--k

\section{Introduction}

The purpose of the modern laser technology is to seek for the simultaneous increase of the laser power and the pulse repetition rate. In order to improve these parameters the power capability of the laser components has to be increased. One of the main components of the laser is the electro-optic modulator, which controls the laser beam polarization. The electro-optic modulators based on the Pockels effect are usually used in the high power lasers. The variation of the light polarization travelling through the Pockels cell is controlled by the electric field, which is produced by the high voltage (HV) pulses applied to the Pockels cell.

Two types of crystal materials are commonly used for the realization of Pockels cells: beta barium borate (BBO) and deuterated potassium dihydrogen phosphate (DKDP). Both electro-optic crystals suffer from the piezoelectric ringing phenomenon [1-7]. It is caused by the short edges $(\approx 6 \mathrm{~ns})$ of high voltage (up to $6.3 \mathrm{kV}$ ) pulses [8] that are required for the operation of the Pockels cell. The piezoelectric ringing phenomenon causes the deformations of the crystal and, as a consequence, the modulation of the contrast ratio $(\mathrm{CR})$. The $\mathrm{CR}$ is the main parameter of the Pockels cell, which presents the ratio of the maximal and minimal intensity of the laser beam in the $Q$-switch operation of the cell. Deformations caused by the piezoelectric ringing

\footnotetext{
* corresponding author; e-mail: giedrius.sinkevicius@ftmc.lt
}

increase the temperature of the crystal due to the frictions. The variation of the crystal temperature may change the polarization angle of the light and the CR.

The investigations of the temperature impact on the polarization of the light of the Pockels cells based on the DKDP crystals have already been performed [9-11]. The results presented in [9-11] show that the temperature change influences the polarization and, as a consequence, the CR.

However, there are no works dedicated to the analysis of the impact of the piezoelectric ringing on the temperature of the $\mathrm{BBO}$ crystal and whether the temperature influences the $\mathrm{CR}$ of the BBO crystal Pockels cells. The purpose of this work is to provide the investigations of these phenomena in the wide frequency range of $\mathrm{HV}$ pulses from $10 \mathrm{kHz}$ to $1 \mathrm{MHz}$.

\section{Experimental setup}

The developed experimental setup is discussed. The block diagram of the optical and electrical part of the setup is presented in Fig. 1. The optical part of the block diagram consists of a diode-pumped solid-state laser, a non-polarizing beam splitter cube, two polarizers (polarizer 1, polarizer 2), a Pockels cell and two detectors (detector 1, detector 2).

The $1064 \mathrm{~nm}$ wavelength laser with the $2 \mathrm{~W}$ of output power and the $1.5 \mathrm{~mm}$ beam diameter was used in the setup as a laser light source. In order to get a measurement reference point to assure the stability of the system, detector 2 was used. The part of the laser's beam intensity gets deflected from the beam splitter into detector 2. The experimental setup allows us to detect the power changes of the laser output. The CR of the Pockels 


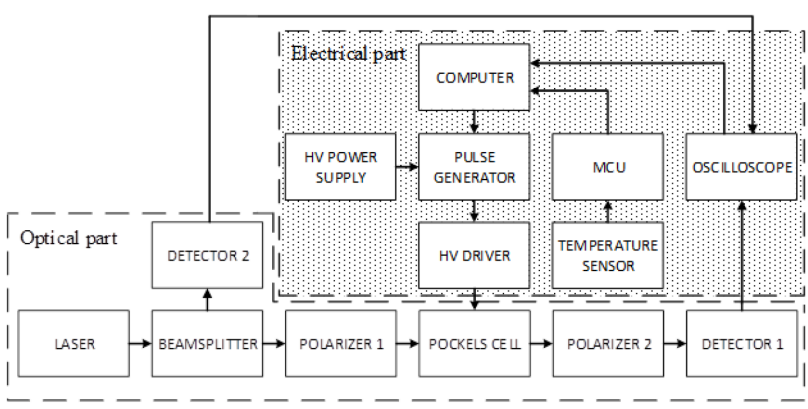

Fig. 1. Block diagram of the experimental setup.

cell was measured with two polarizers, which were placed in front and behind the BBO Pockels cell. The phases of the first and the second polarizers are mutually turned by $90^{\circ}$.

The electrical part of the experimental setup (Fig. 1) consists of the HV power supply, an HV driver, a pulse generator, an oscilloscope, a temperature sensor, a microcontroller unit (MCU) and a computer to control the process of the experiment. Parameters of the HV pulse were controlled via the computer software developed by the authors. The computer sends the data to the pulse generator, which forms control signals for the HV driver operation. The BBO Pockels cell crystal was connected to the output of the HV driver. The signal from the detector 2 was measured with the oscilloscope (Tektronix MDS3014 model) and sent to the computer for the further data processing. MCU reads the value from the temperature sensor and sends it to the computer.

\section{Investigation of the Pockels cell temperature dependence on the frequency}

The measurements of the CR and temperature dependence on the applied HV pulse frequency for 10 different $3 \mathrm{~mm} \times 3 \mathrm{~mm} \times 25 \mathrm{~mm}$ BBO Pockels cell crystals were performed. The piezoelectric ringing phenomenon can be observed by the $1 / \mathrm{CR}$ increase (the dependences of $1 / \mathrm{CR}$ instead of $\mathrm{CR}$ are usually used in the works dedicated to this topic, e.g. [4]). The purpose of the investigation was to fix the frequencies, at which the $1 / \mathrm{CR}$ increases, and to determine whether the BBO Pockels cell crystals temperature increase occurs at these frequencies. Measurements were performed for 10 unique $3 \mathrm{~mm} \times 3 \mathrm{~mm} \times 25 \mathrm{~mm}$ BBO Pockels cell crystals under the same conditions. The temperature sensor was fixed at the side of the BBO crystal. Sensors were electrically isolated from the source of the HV pulses. However, due to the high electromagnetic interference generated during the fast rise and fall of $\mathrm{HV}[12,13]$, the measurement of the temperature was not accurate when the $\mathrm{HV}$ pulses were applied to the BBO Pockels cell crystal. The solution was to measure the temperature when the pulse generator, which controls the HV pulses applied to the Pockels cell, was stopped. The automatic measurement software, which was developed by the authors, was accordingly adjusted to handle this task. Temperature measurements were made every time the HV frequency was changed.

The measurements were recorded while changing the frequency of $\mathrm{HV}$ pulses, which were applied to the BBO Pockels cell crystal. The frequency was changed with the step of $5 \mathrm{kHz}$ every $4 \mathrm{~s}$. The pulse width was adjusted at every step to accomplish the $50 \%$ duty cycle at all frequencies. Two parameters were recorded: the $1 / \mathrm{CR}$ and the crystal temperature. The purpose of this experiment was to determine the frequencies, at which the temperature increase occurs. Therefore, the exact temperature, at which the BBO Pockels cell crystal would operate at each frequency was not determined by this measurement. If the measurement duration were longer the temperature rise would be higher.

The averaged dependences of the $1 / \mathrm{CR}$ and the crystal temperature on the HV pulse frequency obtained using the measurement results of all 10 BBO Pockels cell crystals are presented in Fig. 2. It is seen that the temperature increases when the $1 / \mathrm{CR}$ value increases (piezoelectric ringing occurs).

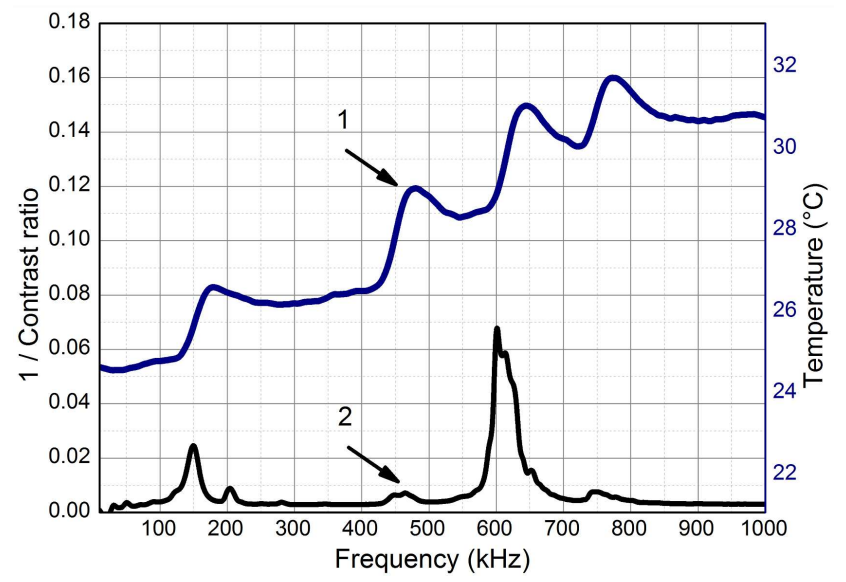

Fig. 2. The averaged dependence of the $1 / \mathrm{CR}$ and temperature of $3 \mathrm{~mm} \times 3 \mathrm{~mm} \times 25 \mathrm{~mm}$ Pockels cell BBO crystals on the applied HV pulse frequency. 1 - Pockels cell crystal temperature, $2-1 / \mathrm{CR}$.

The averaged dependences of the $1 / \mathrm{CR}$ and the crystal temperature on the $\mathrm{HV}$ pulse frequency showing standard deviations, in the $400-800 \mathrm{kHz}$ range for $3 \mathrm{~mm} \times 3 \mathrm{~mm} \times 25 \mathrm{~mm}$ Pockels cell BBO crystals, are given in Fig. 3. The highest standard deviation of the $1 / \mathrm{CR}$ was observed at the $605 \mathrm{kHz} \mathrm{HV}$ pulse frequency. However, there was no rise of the temperature deviation at this frequency. Therefore, it can be stated that the deviation of the $1 / \mathrm{CR}$ and the temperature of the crystal does not correlate with each other. Because of this, the conclusion can be drawn that the crystal temperature rise caused by the piezoelectric ringing phenomenon does not influence the CR of the BBO Pockels cell.

The transients of the BBO Pockels cell temperature were measured at various $\mathrm{HV}$ pulse frequencies at the $50 \%$ duty cycle, i.e. for the situation when the HV 


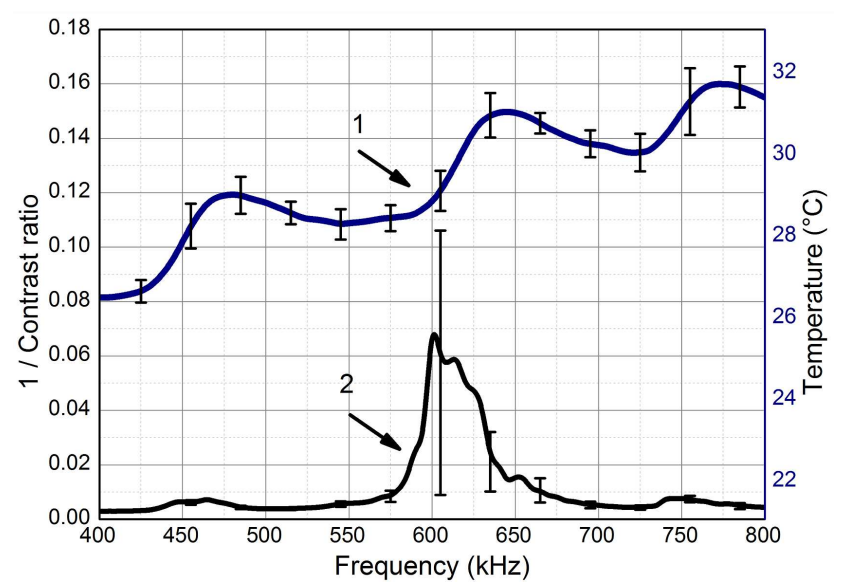

Fig. 3. The averaged dependences and their deviation ranges of $1 / \mathrm{CR}$ and temperature of $3 \mathrm{~mm} \times 3 \mathrm{~mm} \times 25 \mathrm{~mm}$ Pockels cell $\mathrm{BBO}$ crystals on the applied HV pulse frequency. 1 - Pockels cell crystal temperature, $2-1 / \mathrm{CR}$.

pulse duration is equal to half of the period. Four HV pulse frequencies were selected for the measurement: 150 , 350,455 , and $605 \mathrm{kHz}$. Three measurements were performed at frequencies where the piezoelectric ringing phenomenon impact was strongest (the highest $1 / \mathrm{CR}$ increase is observed): 150,455 , and $605 \mathrm{kHz}$. The HV pulse frequency of $350 \mathrm{kHz}$ was selected because at this frequency the piezoelectric ringing does not occur. The measurement results are presented in Fig. 4. It is seen that the heat, which is generated at different frequencies differs. As expected, the lowest $24^{\circ} \mathrm{C}$ temperature was measured at the $350 \mathrm{kHz}$ frequency, at which the piezoelectric ringing does not occur. However, at the HV pulse frequency of $605 \mathrm{kHz}$, where the highest piezoelectric ringing amplitudes occur, the temperature reaches $43{ }^{\circ} \mathrm{C}$. The obtained investigation results prove that the piezoelectric ringing phenomenon increases the temperature of the BBO Pockels cell crystals.

The investigation of the $1 / \mathrm{CR}$ dependence on the temperature for the BBO Pockels cell crystals was performed to find out whether the temperature rise caused by the piezoelectric ringing influences the $1 / \mathrm{CR}$ value. The crystal temperature was varied from 20 to $80^{\circ} \mathrm{C}$ using the oven intended for the investigation of crystals. The obtained dependences are presented in Fig. 5. It is seen that the CR of the BBO Pockels cell crystal practically does not depend on the temperature. Therefore the conclusion can be made that the temperature rise caused by the piezoelectric ringing practically does not influence the $\mathrm{CR}$ of the $\mathrm{BBO}$ Pockels cell crystal. Because of this $\mathrm{BBO}$ crystals does not require the temperature stabilization even while working at frequencies where the piezoelectric ringing occurs.

The DKDP Pockels cell ringing phenomenon was also investigated. High voltage pulse, which was applied to the electrodes of DKDP and BBO Pockels cells, modulate laser beam that travels through the Pockels cell crystal.

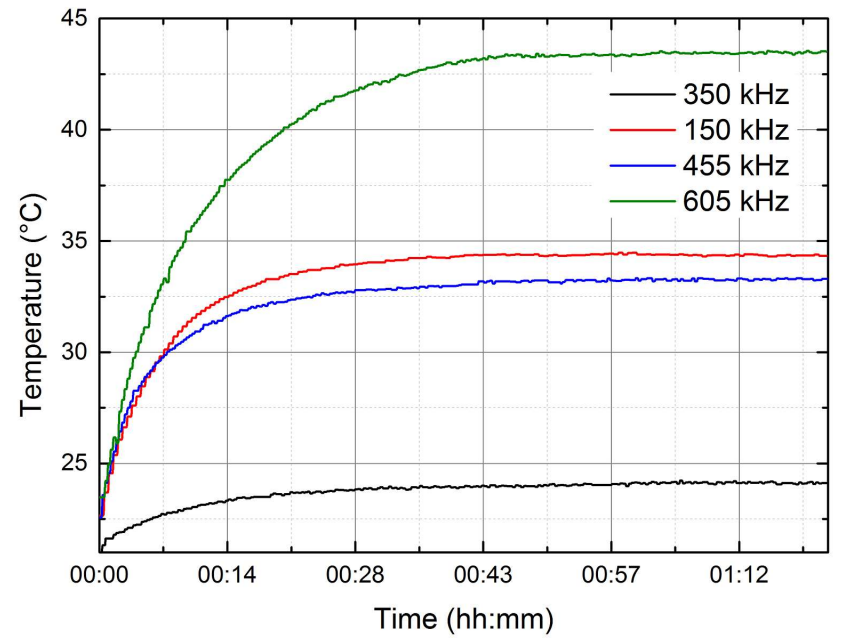

Fig. 4. The temperature transients at various $\mathrm{HV}$ pulse frequencies of the $3 \mathrm{~mm} \times 3 \mathrm{~mm} \times 25 \mathrm{~mm}$ Pockels cell BBO crystal.

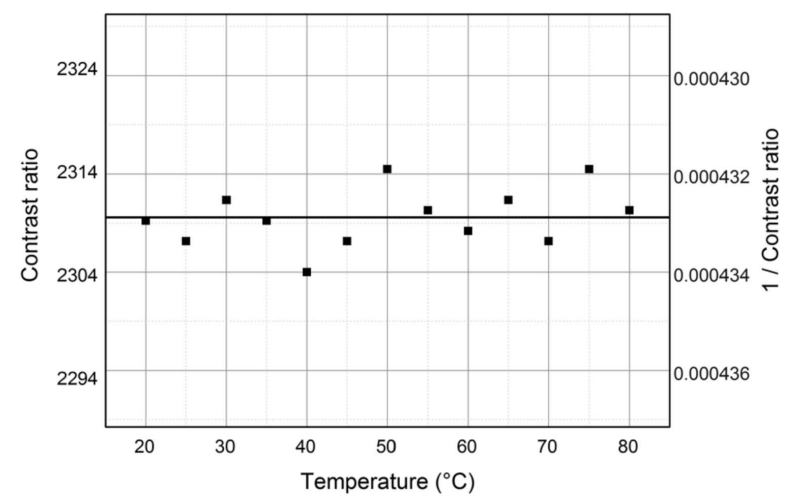

Fig. 5. Dependence of the BBO Pockels cell crystal contrast ratio on the temperature.

The optical modulation oscillograms of DKDP and BBO Pockels cells taken from the detector 1 are presented in Fig. 6. It is seen that the signal of the detector is distorted in the case when the DKDP Pockels cell is used, i.e. the oscillations of $\mathrm{CR}$ caused by the piezoelectric ringing are observed. The piezoelectric ringing is caused by the both (rising and falling) edges of HV pulse. The signal of the detector shows almost none of the piezoelectric ringing phenomenon in the situation when the cell with the BBO crystal is applied.

The ringing of the DKDP Pockels cell occurs even after the first pulse, while the $\mathrm{BBO}$ requires multiple pulse stimulation. High piezoelectric ringing amplitudes produce the heat. It is known that the polarization angle of the light, which travels through the DKDP Pockels cell crystal, depends on the temperature [10, 11]. Consequently, the temperature change causes the variation of the CR as well. Therefore, the DKDP Pockels cell crystals require the active temperature stabilization for the correct operation. The facts mentioned above allow us to conclude that from the point of view of the $\mathrm{CR}$ temper- 


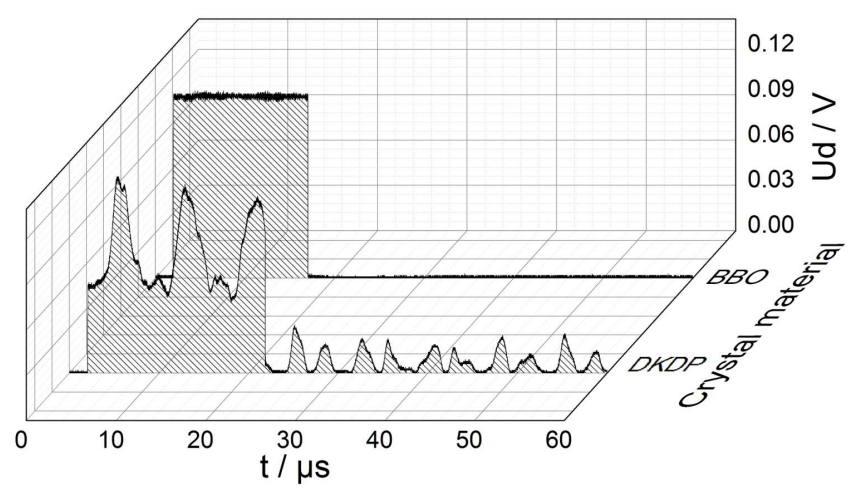

Fig. 6. The Pockels cell modulation oscillograms taken from the first detector.

ature stability Pockels cells based on the BBO crystals are more preferable.

\section{Conclusions}

1. The investigations have proved that the piezoelectric ringing phenomenon increases the temperature of the beta barium borate Pockels cell crystals. The temperature of the crystal reaches $43^{\circ} \mathrm{C}$ in the range of $10 \mathrm{kHz}$ to $1 \mathrm{MHz}$ of the high voltage pulse frequency.

2 . The crystal temperature rise caused by the piezoelectric ringing phenomenon does not influence the contrast ratio of the beta barium borate Pockels cell. Therefore, such crystals do not require the temperature stabilization even when working at high voltage pulse frequencies where the piezoelectric ringing occurs.

3. The Pockels cells based on the beta barium borate crystals are more preferable from the point of view of the contrast ratio temperature stability as compared to the cells with deuterated potassium dihydrogen phosphate.

\section{References}

[1] M. Roth, M. Tseitlin, N. Angert, Glass Phys. Chem. 31, 86 (2005).

[2] H. Lan, F. Liang, Z. Lin, H. Yu, H. Zhang, J. Wang, Int. J. Opt. 63, 694 (2017).

[3] S. Ma, H. Yu, H. Zhang, X. Han, Q. Lu, C. Ma, R. Boughton, J. Wang, Sci. Rep. 6, 30517 (2016).

[4] F. Bergmann, M. Siebold, M. Loeser, F. Röser, D. Albach, U. Schramm, Appl. Sci. 5, 761 (2015).

[5] J. Shang, J. Sun, Q. Li, J. Yang, L. Zhang, J. Xu, Sci. Rep. 7, 4651 (2017).

[6] D. Nickel, C. Stolzenburg, A. Beyertt, A. Geisen, J. Häußermann, F. Butze, M. Leitner, Rev. Sci. Instrum. 76, 033111 (2005).

[7] O. de Vries, T. Saule, M. Plötner, F. Lücking, T. Eidam, J. Limpert, S. Holzberger, T. Schreiber, R. Eberhardt, I. Pupeza, A. Tünnermann, Adv. Solid State Lasers 2015, ATh4A.4 (2015).

[8] S. Mittal, K. Arvind, L. A.S, P. S.S, IETE J. Res. 63, 694 (2017).

[9] V.M. Kedyulich, A.G. Slivka, E.I. Gerzanich, A.M. Guivan, P.M. Lukach, Condens. Matter Phys. 6, 271 (2003)

[10] A. Starobor, O. Palashov, Appl. Opt. 55, 7365 (2016).

[11] Y. Takeuchi, A. Yoshida, S. Tokita, M. Fujita, J. Kawanaka, J. Phys. Conf. Ser. 112, 032061 (2008).

[12] A. Sharma, K.K. Mishra, M.R. Ramaiah, P. Naik, P.D. Gupta, Sadhana - Acad. Proc. Eng. Sci. 32, 235 (2008).

[13] Y. Xu, W. Chen, H. Liang, Y. Li, F. Liang, Q. Shen, S. Liao, C. Peng, AIP Adv. 7, 115210 (2017).

[14] G. Sinkevičius, A. Baškys, Sci. - Future Lith. Moksl. - Liet. Ateitis 9, 308 (2017). 